\title{
The influence of age and body mass index on relative accuracy of energy intake among Japanese adults
}

\author{
Hitomi Okubo ${ }^{1}$, Satoshi Sasaki ${ }^{1, * \dagger}$, Naoko Hirota $^{2}$, Akiko Notsu $^{3}$, Hidemi Todoriki $^{4}$, \\ Ayako Miura ${ }^{5}$, Mitsuru Fukui $^{6}$ and Chigusa Date ${ }^{7} \ddagger$ \\ 'Scientific Evaluation of Dietary Reference Intakes' Project, National Institute of Health and Nutrition, Tokyo, Japan: \\ ${ }^{2}$ Department of Living Sciences, Nagano Prefectural College, Nagano, Japan: ${ }^{3}$ Tottori College, Tottori, Japan: \\ ${ }^{4}$ Department of Environmental and Preventive Medicine Faculty of Medicine, School of Medicine, University of \\ Ryukyus, Okinawa, Japan: ${ }^{5}$ Department of Nutritional Health, Kwassui Women's College, Nagasaki, Japan: \\ ${ }^{6}$ Department of Statistics, Osaka City University Medical School, Osaka, Japan: ${ }^{7}$ Department of Food Sciences and \\ Nutrition, School of Human Environmental Sciences, Mukogawa Women's University, Hyogo, Japan
}

Submitted 4 April 2005: Accepted 20 0ctober 2005

\begin{abstract}
Objective: To examine relationships between the ratio of energy intake to basal metabolic rate (EI/BMR) and age and body mass index (BMI) among Japanese adults. Design: Energy intake was assessed by 4 -day semi-weighed diet records in each of four seasons (16 days in total). The EI/BMR ratio was calculated from reported energy intake and estimated basal metabolic rate as an indicator of reporting accuracy.

Setting: Residents in three areas in Japan, namely Osaka (urban), Nagano (rural inland) and Tottori (rural coastal).

Subjects: One hundred and eighty-three healthy Japanese men and women aged $\geq 30$ years.

Results: The oldest age group ( $\geq 60$ years) had higher EI/BMR values than the youngest age group (30-39 years) in both sexes ( 1.74 vs. 1.37 for men; 1.65 vs. 1.43 for women). In multiple regression analyses, age correlated positively (partial correlation coefficient, $\beta=0.012, P<0.001$ for men; $\beta=0.011, P<0.001$ for women) and BMI correlated negatively $(\beta=-0.031, P<0.001$ for men; $\beta=-0.025, P<0.01$ for women) with EI/BMR.

Conclusion: Age and BMI may influence the relative accuracy of energy intake among Japanese adults.
\end{abstract}

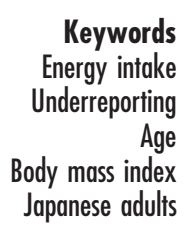

Reliable dietary information plays a critical role in many aspects of human nutrition. Investigators have often relied on self-reported dietary data assessed by diet records, 24hour dietary recalls and food-frequency questionnaires to interpret the associations between diet and disease. However, the results of various studies applying different assessment methods and investigating different populations have shown common problems such as reporting bias $^{1,2}$. In particular, underreporting of energy intake is a serious threat to the validity of self-reported dietary assessment data. Studies using the doubly labelled water technique as an external biomarker of energy intake not only reveal underreporting of energy intake, but also

tCorrespondence address: 1-23-1 Toyama, Shinjuku-ku, Tokyo 1628636, Japan.

$\ddagger$ Present address: Department of Food Science and Nutrition, Faculty of Human Life and Environment, Nara Women's University, Nara, Japan. identify the subject characteristics and factors associated with underreporting ${ }^{3,4}$. Moreover, other studies using the ratio of energy intake to basal metabolic rate (EI/BMR) as an alternative approach to identify the low energy reporters have shown similar results ${ }^{5,6}$.

Most studies found a higher proportion of underreporting among women and older subjects ${ }^{7,8}$. Moreover, underreporting of energy intake was common among obese subjects $^{9-11}$, but was also observed in non-obese subjects $^{12,13}$. Other factors such as body image, health consciousness, social desirability, educational level and smoking status also affected reporting accuracy ${ }^{2,14,15}$. However, all of these studies were conducted in Western countries. The only study conducted in Japan showed a significantly negative correlation between BMI and EI/ BMR among women aged $18-20$ years $^{16}$. Thus the purpose of the present study was to examine the relative accuracy of self-reported energy intake among various age ranges in the Japanese population. 


\section{Subjects and methods}

\section{Subjects}

We selected three areas which have different geographical conditions in Japan: Osaka (urban), Nagano (rural inland) and Tottori (rural coastal). We invited 32 healthy married women aged 30-69 years from each of the three areas to distribute eight women equally in each age class of 30-39, 40-49, 50-59 and 60-69 years. The total number of women recruited was 96. Their husbands (aged 31-76 years) were also invited to participate in the study. None of the subjects was currently receiving or had recently received diet counselling from a doctor or dietitian, nor had a history of educational hospitalisation for diabetes. The subjects were not randomly sampled but asked by local study staff to participate in the study. Here, subject recruitment was continued until a sufficient number of subjects was obtained. Prior to the study, we held group orientations for the subjects where we explained the study purposes and protocol. All subjects giving written informed consent were finally considered eligible for the study.

\section{Dietary assessment}

The subjects completed 4-day semi-weighed diet records four times at 3-month intervals from November 2002 to August 2003. Dietary intake was assessed from four randomly assigned days, including one weekend day and three weekdays. A digital scale (Tanita KD-173; $\pm 2 \mathrm{~g}$ precision for $0-250 \mathrm{~g}$ and $\pm 4 \mathrm{~g}$ precision for $250-1000 \mathrm{~g}$ ) was given to each couple to weigh all the foods eaten. When measurement was difficult, e.g. when eating out, we instructed them to record in as much detail as possible the size and quantity of foods they ate. For each recording day, the subjects were asked to fax the completed forms to the local staff (dietitians). The study staff checked the submitted forms and asked the subjects to add and/or modify the records as necessary by telephone or fax. In some cases, the responses were handed directly to the study staff rather than faxed.

All the collected diet records were checked by trained dietitians in each local centre and then in the study centre. The diet records were analysed for nutrient intake by trained dietitians using the food composition table of Japanese foods, 5 th edition ${ }^{17}$.

\section{Physical activity level and anthropometric measurements}

Physical activity level was obtained from a questionnaire which queried information about each subject's occupation and leisure-time activity. One answer was chosen from four categories, i.e. 'low', 'relatively low', 'moderate' and 'heavy' physical activity level. This classification was referenced to the recommended dietary allowance for Japanese, 6th edition $^{18}$. The gross energy expenditure of each category was considered to require 1.3, 1.5, 1.7 and
1.9 times the BMR, respectively ${ }^{18}$. Therefore, we converted the categorical classification of physical activity level to the ratio of BMR based on above values, and expressed as it as a score for easy interpretation.

Body weight and height were measured to the nearest $0.1 \mathrm{~kg}$ and $0.1 \mathrm{~cm}$, respectively, with subjects wearing light clothing and no shoes. BMI was calculated as body weight (kg) divided by the square of body height $\left(\mathrm{m}^{2}\right)$. We classified BMI into four categories: $<18.5 \mathrm{~kg} \mathrm{~m}^{-2}, 18.5-$ $24.9 \mathrm{~kg} \mathrm{~m}^{-2}, 25.0-27.9 \mathrm{~kg} \mathrm{~m}^{-2}$ and $\geq 28 \mathrm{~kg} \mathrm{~m}^{-2}$. Because the proportion of obese subjects (BMI $\geq 30 \mathrm{~kg} \mathrm{~m}^{-2}$ ) was very low ( $n=1$ for men aged 40-49 years; $n=0$ for women), BMI $\geq 28 \mathrm{~kg} \mathrm{~m}^{-2}$ was used as the highest category instead of $\geq 30 \mathrm{~kg} \mathrm{~m}^{-2}$ in the present analysis.

BMR was estimated for each subject using formulas based on body weight given by the Food and Agriculture Organization/World Health Organization/United Nations University (FAO/WHO/UNU) ${ }^{19}$ as follows.

- Men aged 30-60 years: $\mathrm{BMR}=0.0485 \times$ bodyweight $(\mathrm{kg})+3.67$.

- Men aged $>60$ years: $\mathrm{BMR}=0.0565 \times$ bodyweight $(\mathrm{kg})+2.04$.

- Women aged 30-60 years: $\mathrm{BMR}=0.0364 \times$ bodyweight $(\mathrm{kg})+3.47$.

- Women aged $>60$ years: $\mathrm{BMR}=0.0439 \times$ bodyweight $(\mathrm{kg})+2.49$.

\section{Statistical analysis}

We included 183 subjects (91 women and 92 men) with complete 16-day diet records living in the Osaka (29 women and 30 men), Nagano (31 women and 31 men) and Tottori ( 31 women and 31 men) areas in the present analysis.

We calculated the ratio EI/BMR to evaluate the relative accuracy of the reported energy intake. Subjects were allocated into quintiles of EI/BMR to compare 'low energy reporters' with 'high energy reporters'. Low ratios describe subjects reporting comparatively low energy intake relative to their energy requirement. To compare the relative degree of under- and overreporting, we temporarily used the values defined by $\mathrm{FAO} / \mathrm{WHO} / \mathrm{UNU}$ : the minimum survival level of 1.27 , the sedentary level for men of 1.55 and women of 1.56, and the maximum sustainable lifestyle level of 2.0-2.4.

Results are given as mean \pm standard deviation. Student's $t$-test and one-way analysis of variance (ANOVA) were used to test for differences between the groups. When ANOVA indicated a difference among the groups, Dunnett's $t$-test was applied to compare to the first group as a control. The chi-square test was used to test for proportionate differences between categories. Multivariate evaluation of the simultaneous effects of age, BMI, physical activity level and living area on EI/BMR was performed by a stepwise multiple regression analysis. 
Factors affecting self-reported energy intake

We also computed the partial correlation coefficients between each independent variable and EI/BMR adjusting for other independent variables.

All statistical analyses were performed using version 8.2 of the SAS software package (SAS Institute, Inc., Cary, NC, USA). A $P$-value of $<0.05$ was considered significant.

\section{Results}

Table 1 presents a summary of the physical characteristics of the subjects. Mean age was $52.8 \pm 12.1$ (range 31-76) years in men and $49.5 \pm 11.4$ (range 31-69) years in women. Mean values of EI/BMR were not different between sexes (1.55 for men vs. 1.48 for women, $P=0.12$ ). Men had a higher BMI (23.3 vs. $22.1 \mathrm{~kg} \mathrm{~m}^{-2}$, $P<0.01)$ and a higher proportion of overweight (21\% vs. $11 \%$ for BMI of $25.0-27.9 \mathrm{~kg} \mathrm{~m}^{-2}$ and $10 \% \mathrm{vs.} 2 \%$ for BMI $\left.\geq 28 \mathrm{~kg} \mathrm{~m}^{-2}, P=0.03\right)$ than women. Men had a higher physical activity level than women (1.48 vs. 1.43 , $P=0.02$ ), and $38 \%$ and $59 \%$ of women were classified into low and relatively low physical activity levels, respectively.

Table 2 presents a summary of the physical characteristics of men and women in the four age groups (30-39, 40-49, 50-59 and $\geq 60$ years). Body height decreased with increasing age in both sexes. Body weight and BMR increased as age increased to 40-49 years, and then decreased with increasing age group in both sexes. Although BMI was lowest among the youngest age group in both sexes, a statistically significant difference between age groups was observed only for women $(P<0.01)$. Energy intake was not different between age groups in either sex. On the other hand, mean EI/BMR became significantly higher with increase in age for men

Table 1 Characteristics of study subjects* $(n=183)$

\begin{tabular}{|c|c|c|c|}
\hline & Men $(n=92)$ & Women $(n=91)$ & $P$-value $\dagger$ \\
\hline Age (years) & $52.8 \pm 12.1$ & $49.5 \pm 11.4$ & 0.06 \\
\hline Body height $(\mathrm{cm})$ & $168.0 \pm 6.7$ & $155.6 \pm 5.9$ & $<0.001$ \\
\hline Body weight (kg) & $66.2 \pm 11.2$ & $53.4 \pm 7.2$ & $<0.001$ \\
\hline Reported El (MJ day ${ }^{-1}$ ) & $9.9 \pm 1.8$ & $7.8 \pm 1.2$ & $<0.001$ \\
\hline BMR (MJ day $\left.{ }^{-1}\right) \ddagger$ & $6.5 \pm 0.9$ & $5.3 \pm 0.4$ & $<0.001$ \\
\hline $\mathrm{El} / \mathrm{BMR}$ & $1.55 \pm 0.31$ & $1.48 \pm 0.24$ & 0.12 \\
\hline $\mathrm{BMI}\left(\mathrm{kg} \mathrm{m}^{-2}\right)$ & $23.3 \pm 3.1$ & $22.1 \pm 2.6$ & $<0.01$ \\
\hline$<18.5$ & $4(4)$ & $6(7)$ & $0.03 \S$ \\
\hline $18.5-24.9$ & $60(65)$ & $73(80)$ & \\
\hline $25.0-27.9$ & $19(21)$ & $10(11)$ & \\
\hline$\geq 28.0$ & $9(10)$ & $2(2)$ & \\
\hline Physical activity level & $1.48 \pm 0.19$ & $1.43 \pm 0.11$ & 0.02 \\
\hline Low & $37(40)$ & $35(38)$ & $<0.001 \S$ \\
\hline Relatively low & 36 (39) & $54(59)$ & \\
\hline Moderate & $11(12)$ & $2(2)$ & \\
\hline Heavy & $8(9)$ & $0(0)$ & \\
\hline
\end{tabular}

EI - energy intake; BMR - basal metabolic rate; BMI - body mass index. * Values are expressed as mean \pm standard deviation or $n(\%)$. † Significant difference between sexes ( $t$-test).

$\ddagger$ BMR was calculated using formulas given by the Food and Agriculture Organization/World Health Organization/United Nations University $(1985)^{19}$

$\S$ Significant difference between sexes in all categories (chi-square test).

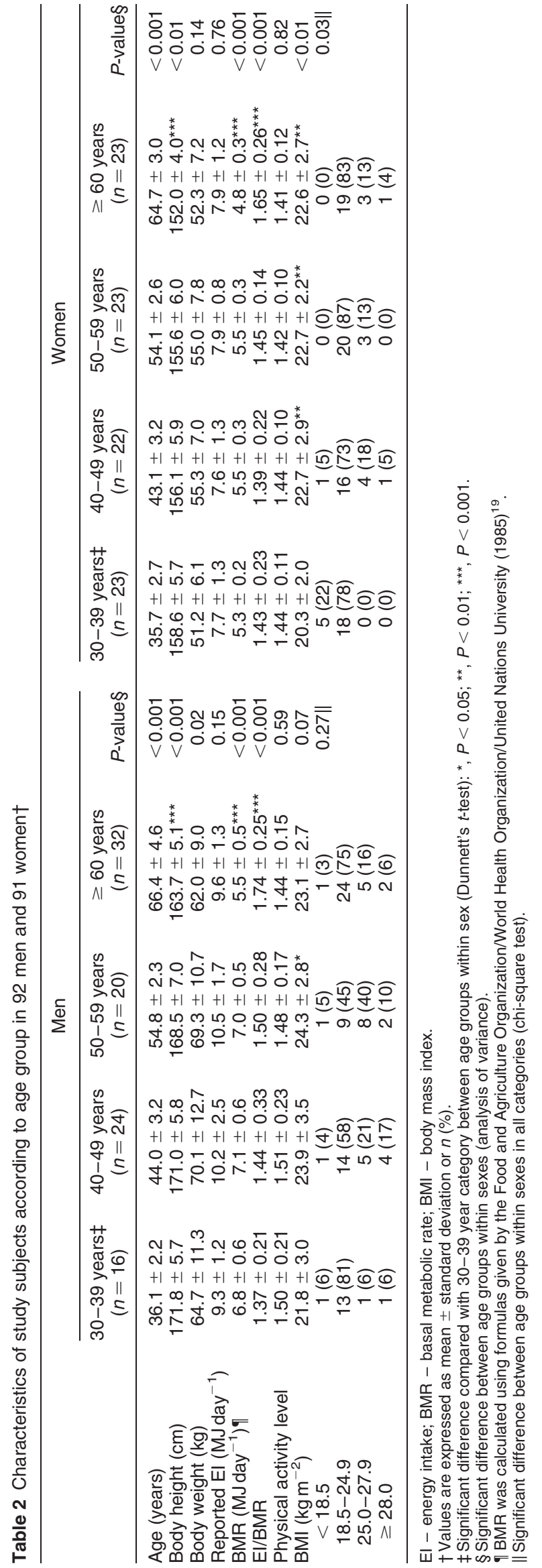


$(P<0.001)$. Although women aged $40-49$ years had the lowest EI/BMR among the women, the trend of the relationship between mean EI/BMR and age was almost the same as that of men $(P<0.001)$.

Table 3 presents the mean values of anthropometric characteristics by quartile of EI/BMR. Age and reported energy intake increased significantly with the increase in EI/BMR in both sexes (all $P<0.001$ except for age in women, where $P<0.01)$. However, with increasing EI/ BMR quartile, body height and body weight decreased significantly in men (both $P<0.01$ ), as did BMR in both sexes ( $P<0.001$ for men, $P<0.01$ for women). BMI was slightly lower in the lowest category of EI/BMR than in the other categories in men, although it was not significant.

Table 4 shows the results of multiple regression analyses with $\mathrm{EI} / \mathrm{BMR}$ as the dependent variable to examine the prediction for relative accuracy of reporting. For men, age and physical activity level correlated positively (partial regression coefficient, $\beta=0.012, \quad P<0.001$ and $\beta=0.377, P=0.01$, respectively), and BMI and living area (urban) correlated negatively $(\beta=-0.031$, $P<0.001$ and $\beta=-0.114, P=0.045$, respectively), with EI/BMR. On the other hand, age and body height correlated positively $(\beta=0.011, P<0.001$ and $\beta=0.011$. $P=0.01$, respectively) and BMI correlated negatively $(\beta=-0.025, P<0.01)$ with EI/BMR for women. All the independent variables explained $35.7 \%$ and $25.7 \%$ of the variation in $\mathrm{EI} / \mathrm{BMR}$ for men and women, respectively.

Figures $1 \mathrm{a}$ and $1 \mathrm{~b}$ show the joint effect of age and BMI on EI/BMR values by cross-classifying subjects by both variables. Compared with subjects classified into the lowest BMI and oldest age group, subjects in the highest

Table 4 Results of stepwise multiple regression analyses with El/BMR ratio as dependent variable*

\begin{tabular}{lrccc}
\hline Independent variable & $\beta \dagger$ & SE $\ddagger$ & $P$-value & $\begin{array}{c}\text { Partial } R^{2} \\
(\%) \S\end{array}$ \\
\hline Men $(n=92)$ & \multicolumn{5}{c}{} \\
Age (years) & 0.012 & 0.002 & $<0.001$ & 17.9 \\
BMI (kg m ${ }^{-2}$ ) & -0.031 & 0.009 & $<0.001$ & 9.9 \\
Physical activity level & 0.377 & 0.145 & 0.01 & 4.8 \\
Living area (rural coastal area as reference) & & \\
$\quad$ Urban & -0.114 & 0.056 & 0.05 & 3.1 \\
Women ( $n=91)$ & & & & \\
Age (years) & 0.011 & 0.002 & $<0.001$ & 12.1 \\
BMI (kg m ${ }^{-2}$ ) & -0.025 & 0.009 & 0.005 & 7.0 \\
Body height (cm) & 0.011 & 0.004 & 0.01 & 6.6 \\
\hline
\end{tabular}

EI - energy intake; BMR - basal metabolic rate; BMI - body mass index. ${ }^{*}$ Age (as a continuous variable), BMI (as a continuous variable), height (as a continuous variable), physical activity level (as a continuous variable) and area of living (rural coastal, rural inland, urban) were entered into the model as independent variables.

†Partial regression coefficient; change in the dependent variable related to a one-unit change in the independent variable.

$\ddagger$ Standard error of the regression coefficient.

$\S$ Explained variance; adjusted $R^{2}$ and $P$-values are for independent variables in multiple regression analysis. $R^{2}$ value for EI/BMR was $35.7 \%$ and $25.7 \%$ for men and women, respectively, when all variables were included in the model. 

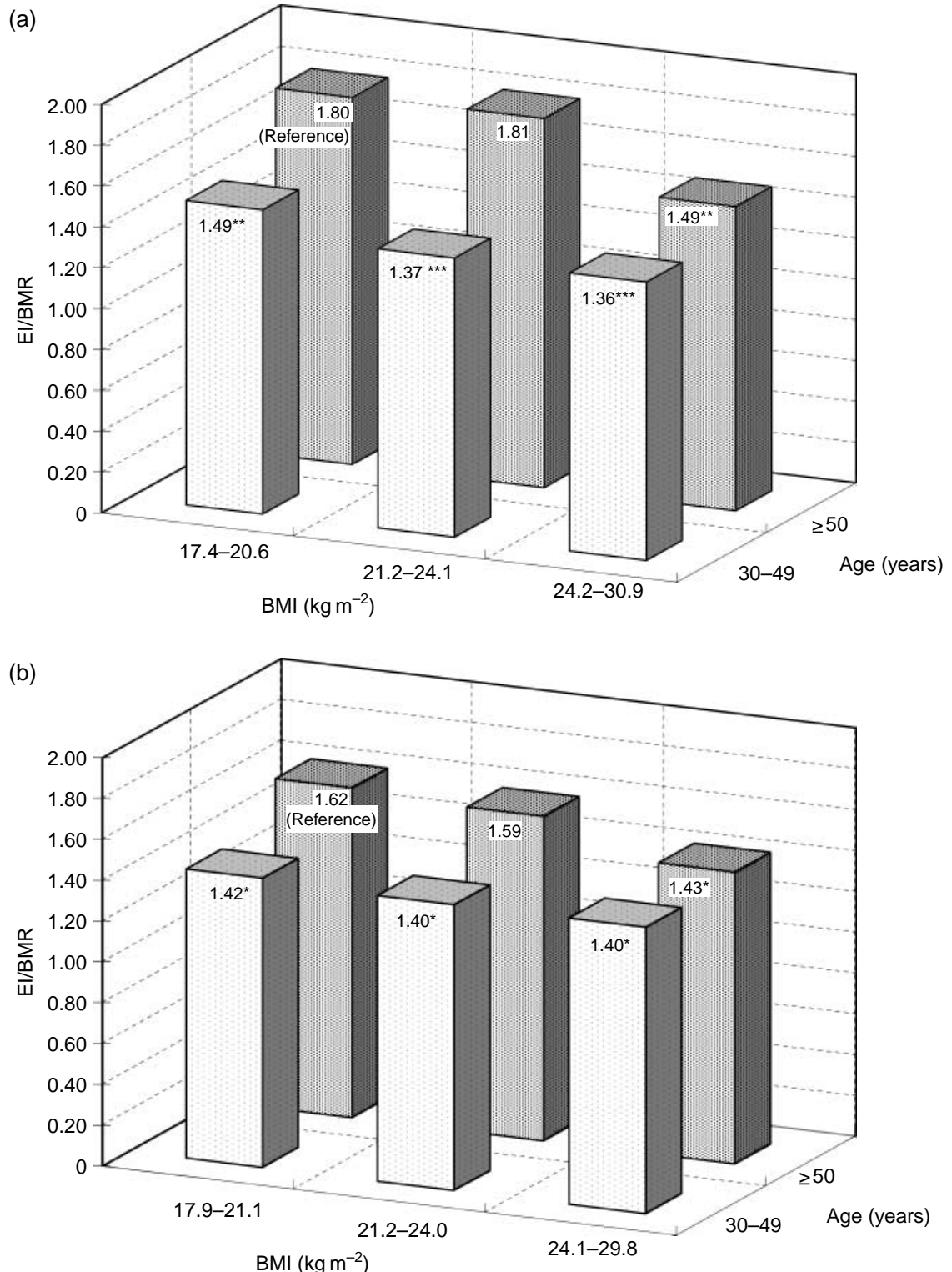

Fig. 1 The interaction of age and body mass index (BMI) in relationships with the ratio of reported energy intake to estimated basal metabolic rate (EI/BMR). Mean value of EI/BMR by tertile of BMI and age group (30-49, $\geq 50$ years) in (a) Japanese men aged $32-76$ years $(n=92)$ and (b) Japanese women aged 31-69 years $(n=91)$. El/BMR values were adjusted for physical activity level and living area. Significance of difference compared with the oldest age and lowest BMI group (Dunnett's $t$-test of one-way analysis of variance): ${ }^{\star}, P<0.05 ;{ }^{* \star}, P<0.01 ;{ }^{* * \star}, P<0.001$

BMI and youngest age group had EI/BMR that was $24 \%$ and $14 \%$ lower in men and women, respectively.

\section{Discussion}

To our knowledge, this is the first report to evaluate $\mathrm{EI} / \mathrm{BMR}$ values over a wide age range of Japanese men and women. We conducted semi-weighed diet records for 4 days in four seasons, which is often considered to be the most accurate and precise method for determining energy intake. Furthermore, fax delivery was used so that we could check the diet records immediately on each survey day. Therefore, we believe that the data have higher precision than in any other such survey conducted in Japan. The EI/BMR in our study was 1.55 among men and 1.48 among women. Although we refrained from using a specific cut-off value to identify underreporters, 20\% and $23 \%$ of men and women, respectively, showed EI/BMR below 1.27, the minimum survival level reported by $\mathrm{FAO} / \mathrm{WHO} / \mathrm{UNU}^{19}$. Moreover, the proportion of subjects with EI/BMR $<1.27$ decreased with increasing age in both sexes, except in the 40-49 year age group in women. However, $10 \%$ and $4 \%$ of men and women, respectively, showed EI/BMR exceeding 2.0 as the maximum level. Even when physical activity level was considered, the proportion of subjects with EI/BMR $>2.0$ increased with 
increasing age, and was especially more pronounced in the age group $\geq 60$ years for both sexes. This indicates that older Japanese men and women tend to relatively overestimate energy intake rather than underreport.

The main finding of this study was that age and BMI independently affect EI/BMR as a positive and a negative factor, respectively. The statistical power of these findings became stronger after adjustment for potentially confounding factors such as physical activity level and living area (urban or rural) for both sexes (Figs 1a and 1b). According to previous studies, physiological and psychological factors are also related to reporting accuracy; for example, smoking habits, education level, socio-economic status and obesity-related behaviours ${ }^{14,15,20-22}$. However, we did not examine the effect of these factors on reporting accuracy because of a lack of information.

Most studies conducted in Western countries revealed that underreporting of energy intake was more prevalent among older subjects than among younger counterparts $7,23,24$. The tendency was completely opposite in this Japanese population. To our knowledge, no previous study has found underreporting to be more prevalent among younger compared with older subjects, either in Western or Asian countries. Possible factors affecting reporting accuracy may include dietary consciousness and knowledge of foods and diet. According to the National Nutrition Survey in Japan ${ }^{25}$, the percentage of subjects who paid high attention to diet and nutrition was $12.1 \%, 17.5 \%, 24.4 \%$ and $27.2 \%$ among 30-39-, 40-49-, 50-59- and $\geq 60$-year-old men, respectively, and $27.5 \%, 35.7 \% 42.9 \%$, and $48.6 \%$, respectively, among women. The capability to recognise foods and diet may be related to recording as correctly as possible. Some previous studies reported that cultural, behavioural and psychological factors affect reporting accuracy $^{14,15,20-22}$. The results were, however, inconsistent and differed among the populations examined. Further research focusing on dietary consciousness and behaviours connected with food and the process of dietary assessment is needed.

Our study has several limitations. First, the subjects may not be representative because they were not randomly sampled from the general Japanese population. Moreover, the participants might be highly health-conscious because almost all of them completed the study despite the strict study design. Second, the sample size was relatively small. Therefore, the results may arise by chance. Third, we cannot exclude the possibility that the subjects changed their dietary behaviour or food choices during the recording periods. However, the relationships between EI/BMR and age and body weight did not change materially when the dietary record data of the first four days were used in the analysis (data not shown). Fourth, we used body height to take into consideration body size although body height is not an ideal marker of body size. Fifth, the reliability of the BMR prediction from the
$\mathrm{FAO} / \mathrm{WHO} / \mathrm{UNU}$ formulas may be inappropriate when applied to the Japanese population ${ }^{26}$. The validity of the self-reported physical activity levels from the 6th Japanese recommended dietary allowance is questionable because of the lack of a validation study ${ }^{18}$.

In summary, the results of the present study suggest that age and BMI may influence the relative accuracy of reported energy intake among Japanese adults. The positive correlation found between age and EI/BMR was especially interesting because almost all previous studies conducted in Western populations showed a negative correlation. This indicates that the factors related to reporting accuracy of energy intake may depend on population characteristics. Further studies are needed to examine whether or not this is a consistent tendency in Asian or Japanese populations.

\section{References}

1 Black AE, Cole TJ. Biased over- or under-reporting is characteristic of individuals whether over time or by different assessment methods. Journal of the American Dietetic Association 2001; 101: 70-80.

2 Livingstone MB, Black AE. Markers of the validity of reported energy intake. Journal of Nutrition 2003; 133(Suppl 3): 895S-920S.

3 Hill RJ, Davies PS. The validity of self-reported energy intake as determined using the doubly labelled water technique. British Journal of Nutrition 2001; 85: 415-30.

4 Trabulsi J, Schoeller DA. Evaluation of dietary assessment instruments against doubly labeled water, a biomarker of habitual energy intake. American Journal of Physiology. Endocrinology and Metabolism 2001; 281: E891-9.

5 Goldberg GR, Black AE, Jebb SA, Cole TJ, Murgatroyd PR, Coward WA, et al. Critical evaluation of energy intake data using fundamental principles of energy physiology. 1. Derivation of cut-off values to identify under-recording. European Journal of Clinical Nutrition 1991; 45: 569-81.

6 Black AE, Goldberg GR, Jebb SA, Livingstone MBE, Cole TJ, Prentice AM. Critical evaluation of energy intake data using fundamental principals of energy physiology: 2. Evaluating the results of published surveys. European Journal of Clinical Nutrition 1991; 45: 583-99.

7 Johansson L, Solvoll K, Bjørneboe G-EA, Drevon CA. Underand overreporting of energy intake related to weight status and lifestyle in a nationwide sample. American Journal of Clinical Nutrition 1998; 68: 266-74.

8 Johnson RK, Goran MI, Poehlman ET. Correlates of overand underreporting of energy intake in healthy older men and women. American Journal of Clinical Nutrition 1994; 59: 1286-90.

9 Buhl KM, Gallagher D, Hoy K, Matthews DE, Heymsfield SB Unexplained disturbance in body weight regulation diagnostic outcome assessed by doubly labeled water and body composition analyses in obese patients reporting low energy intakes. Journal of the American Dietetic Association 1995; 95: 1393-400.

10 Braam LA, Ocke MC, Bueno-de-Mesquita HB, Seidell JC. Determinants of obesity-related underreporting of energy intake. American Journal of Epidemiology 1998; 147: 1081-6

11 Fogelholm M, Männisto S, Vartiainen E, Pietinen P. Determinants of energy balance and overweight in Finland 1982 and 1992. International Journal of Obesity and Related Metabolic Disorders 1996; 20: 1097-104. 
12 Kretsch MJ, Fong AK, Green MW. Behavioral and body size correlates of energy intake underreporting by obese and normal-weight women. Journal of the American Dietetic Association 1998; 99: 300-6.

13 Asbeck I, Mast M, Bierwag A, Westenhofer J, Acheson KJ, Muller MJ. Severe underreporting of energy intake in normal weight subjects: use of an appropriate standard and relation to restrained eating. Public Health Nutrition 2002; 5: $683-90$

14 Tooze JA, Subar AF, Thompson FE, Troiano R, Schatzkin A, Kipnis V. Psychosocial predictors of energy underreporting in a large doubly labeled water study. American Journal of Clinical Nutrition 2004; 79: 795-804.

15 Johansson G, Wikman A, Ahren AM, Hallmans G, Johansson I. Underreporting of energy intake in repeated 24-hour recalls related to gender, age, weight status, day of interview, educational level, reported food intake, smoking habits and area of living. Public Health Nutrition 2001; 4: 919-27.

16 Okubo H, Sasaki S. Underreporting of energy intake among Japanese women aged 18-20 years and its association with reported nutrient and food group intakes. Public Health Nutrition 2004; 7: 911-7.

17 Science and Technology Agency. Standard Tables of Food Composition in Japan, 5th revised ed. Tokyo: Printing Bureau, Ministry of Finance, 2000 [in Japanese].

18 Ministry of Health and Welfare. Recommended Dietary Allowance for Japanese: Dietary Reference Intakes, 6th revised ed. Tokyo: Ministry of Health and Welfare, 1999 [in Japanese].

19 Food and Agriculture Organization/World Health Organization/United Nations University (FAO/WHO/UNU). Energy and Protein Requirements. Report of a Joint
FAO/WHO/UNU Expert Consultation. Technical Report Series No. 724. Geneva: WHO, 1985.

20 Taren DL, Tobar M, Hill A, Howell W, Shisslak C, Bell I, et al. The association of energy intake bias with psychological scores of women. European Journal of Clinical Nutrition 1999; 53: 570-8.

21 Hebert JR, Clemow L, Pbert L, Ockene IS, Ockene JK. Social desirability bias in dietary self-report may compromise the validity of dietary intake measures. International Journal of Epidemiology 1995; 24: 389-98.

22 Kant AK. Interaction of body mass index and attempt to lose weight in a national sample of US adults: association with reported food and nutrient intake, and biomarkers. European Journal of Clinical Nutrition 2003; 57: 249-59.

23 Briefel RR, Sempos CT, McDowell MA, Chien S, Alaimo K. Dietary methods research in the third National Health and Nutrition Examination Survey: under-reporting of energy intake. American Journal of Clinical Nutrition 1997; 65: S1203-9.

24 Horner NK, Patterson RE, Neuhouser ML, Lampe JW, Beresford SA, Prentice RL. Participant characteristics associated with errors in self-reported energy intake from the Women's Health Initiative food-frequency questionnaire. American Journal of Clinical Nutrition 2002; 76: 766-73.

25 Ministry of Health and Welfare. Kokumin Eiyou no Genjou [Annual Report of the National Nutrition Survey in 1998]. Tokyo: Ministry of Health and Welfare, 2000; 45-6 [in Japanese].

26 Yamamura C, Kashiwazaki H. Factors affecting the postabsorptive resting metabolic rate of Japanese subjects: reanalysis based on published data. Japanese of Journal of Nutrition 2002; 60: 75-83 [in Japanese]. 